\title{
A Study of Performance Enhancement in Heterogeneous Multi-Cluster System
}

\author{
Mohit Angurala \\ Assistant Professor \\ CSE \\ Golden College of Engineering \& Technology, \\ Gurdaspur (PB)
}

\author{
Jyoti Bala \\ M.Tech Student \\ CSE \\ Golden College of Engineering \&Technology, \\ Gurdaspur, PB
}

\begin{abstract}
In this system composed of number of clusters of processors. In Parallel Processing, Heterogeneous Scheduling plays main role. BPU is used to compute the speed of processors. Speed heterogeneity is a most important feature which affects the overall performance of the system. The scheduling process used technique of Job scheduling and Processor allocation are two main area of concern for improving the performance in Multi-cluster systems by using parameter of response time that could enhanced the CPU performance in a heterogeneous multi-cluster system. Job sequences decide by Job Scheduling for processor allocation and processor allocation is concerned with assign number of processors for incoming job. In this paper we try to propose a processor allocation job scheduling algorithm that maximizes the CPU utilizations which consists of heterogeneous Multi-cluster System. Scheduling is the most essential part in Multi-cluster System. In Multi-cluster, Multi-cluster schedulers mustn't only for nodes resources allocation but also inter-cluster network utilization. The idea of clustering the jobs based on arrival time. In this paper we produce the value of different parameters to make effective scheduling by job co-allocation in a multi-cluster system. To study the effect of parameters, calculate response waiting time and co-allocation time.
\end{abstract}

\section{General Terms}

CPU Utilization improve

\section{Keywords}

Global scheduler, job sizes, heterogeneous multi-cluster system, scheduling, processor requirement and allocation, FCFS

\section{INTRODUCTION}

Parallel computing could be the simultaneous usages of multiple compute resources to resolve a computational problem:

1) An problem is broken into separate part which will be resolved at same time

2) Each part is further broken right down to a series of instructions

3) Instructions from every part execute continuously on various processor

The performance of a different type of computer system is outlined by three factors.

1) Time to execute a program is amount of instructions to execute.

2) The average number of clock cycles needed.
3) Clock Cycle Time required.

\section{Characteristics of a Parallel System}

A parallel processing system has these characteristics:

1) Each processor in a method can perform tasks concurrently.

2) Tasks may require to be synchronized.

3) Nodes usually share resources, such as for example data, disks, and other devices.

\section{Job Scheduling}

Job scheduling determines the sequence of starting execution of the jobs waiting in the queue.

The scheduling should be done on Online System with symmetric processing stations, Throughput, response time. Therefore, there is a require of scheduler to manage several jobs in the queue.

\section{1) First Come First Served (FCFS):}

In this approach first incoming job request get the highest priority. The first job is checked whether it fits the available resources. If the first job fits the available resources it is executed immediately but if the number of available processors cannot meet the requirement of the first job in queue, all the other jobs would be blocked until enough processors for first job become available and job waits until the next re-scheduling action takes place.

\section{FCFS have Number of advantage and disadvantage:}

\section{Advantage:}

a. Simple

b. Fair.

\section{Disadvantages:}

1. WT dependent on AT

2. Short process struck long process that is the convey effect

3. This is also known as head of blocking 


\section{MULTI-CLUSTER}

It consists of different independent clusters or nodes. Each contribute site is a heterogeneous parallel computer. Every node is heterogeneous in the intelligence that number of processors as well as compute speed of every processor may raises. The sites are linked to each other by interconnection network. A cluster is a consists of number of loosely coupled computers that work closely together as a single computational facility. Clusters are arranged in the way so that the speed and reliability given by single computer can be improved. They are economically compared by the single computer. Clusters combination of hundreds and thousands of standard CPUs

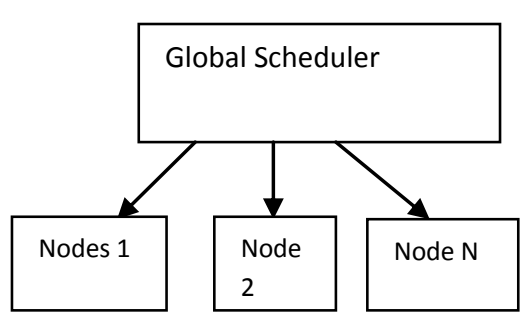

\begin{tabular}{|l|l|}
\hline Nodes & BPUS \\
\hline & \\
& \\
\hline
\end{tabular}

This paper consists of a part as follows: The second part presents the related work. The third is focuses on the proposed policy. The four, in result and. part five talks about conclusion and future work.

\section{RELATED WORK}

In this we make a short overview of most popular Scheduling in Multi-cluster methods. But we mainly focus on the functional aspects of the methods. A brief summary of the literature is given below:

A.C.Kaladevi et al. (2012) [1] it works on Advanced Processor Reservation Algorithm based on priority of jobs. Job may need co-allocation in Multi-cluster system i.e. continuous allocation of resources such as processor in multiple nodes (clusters) which jobs may need low run time that because they more no of resources. It's have main challenging in Processor Allocation and performance raised by large number of requests using priority

Dinesh Gupta et al. (2012) [2] it research regarding different scheduling algorithm to enhanced CPU utilization. Various scheduling Algorithm have different properties which work on different scheduling critics. In given number of Processors, SJF gives minimum average waiting time. The Round Robin improves average response time. In this paper give response time mostly zero and improve I/O efficiency.

Hector Blanco et al. (2011) [6] it focuses on Multi-cluster environments are contains of larger clusters of nodes that act collaboratively, and that allowing computational problems to be treated that need more resources than that available in one single cluster. However, the amount of complexity of the scheduling process is greatly raised by the heterogeneity of resources and co-allocation process that contributes the tasks of parallel jobs across cluster boundaries. His work presents a new scheduling strategy which allocates multiple jobs from the system queue continuously on a heterogeneous multicluster, by applying co-allocation when is essential. His techniques are made-up by a job selection function and a linear programming model to find the best allocation for multiple jobs. The proposed scheduling technique is reduce the execution times of the parallel jobs and the overall response times by $38 \%$ compared with other scheduling strategy in the literature.

Jaehwan Lae et al. (2010) [11] work is based on Scheduling of jobs is an in grid. Scheduling of jobs is main challenge problem in grid. Efficient job scheduling is needed for the effective utilization of the resources. It proposes a grid model as a combination of clusters. In this survey, its apply Divisible Load Theory (DLT) and Least Cost Method (LCM) to model the grid scheduling problem involving multiple worker nodes in each cluster. It proposes a hybrid job scheduling algorithm which minimizes the overall processing time of the job in a grid system that made up of heterogeneous hosts. It shows results show that the proposed algorithm is feasible and improves the makespan considerably.

John Ngubiri et al. (2007) [12] evaluated the performance of co-allocation in multi-cluster system and observed that (i)coallocation is viable if the execution time penalty caused is low; (ii) due to possible heterogeneous communication pattern, co-allocation may not be as viable.

K.C. Huang et al. $(\mathbf{2 0 1 3 , 2 0 1 3 )}[\mathbf{9 , 1 0 ]}$ research regarding adaptive processor allocation based on moldable property of parallel jobs for heterogeneous system and studied about speed of heterogeneous system at inter cluster level in which nodes on different cluster may have different compute speed. But nodes on same site have same speed.

\section{PROPOSED POLICY}

When we start my process with multiple jobs firstly global scheduler check all incoming jobs are met with processor xi . submit job ji to processor xi after job submission scheduling session started. Jobs ji selected with suitable BPUs processor xi (Basic processing units). If ji<xi available if say yes then perform site selection and if no then choose either option CA and WA and calculate parameters.

If job ji with required processor xi done successfully then calculate parameters and if no then scheduling session started again and process continuous as on.

\section{EXPERIMENTAL RESULTS}

This section contains experimental results of proposed work. The overall section contains the response time in waiting, response time in co-allocation. The proposed methodology depicts the concept of clustering the jobs based on arrival time. During the time of scheduling the traditional methods such as First Come First Serve. Multi-cluster are systems are use now a days to obtain high performance and high system utilizations.

\section{Performance Parameters}

The performance of algorithms is usually measured using performance enhanced parameters such average response time (waiting time), average response time (co-allocation)

Response time (Waiting)-

Typically, Utilization and Throughput are traded off for better Response Time. Response time is important for OS's that aim to be user-friendly. In general, we would like to optimize the average measure. In some cases, minimum and maximum values are optimized, e.g. it might be a good idea to minimize the maximum response time 


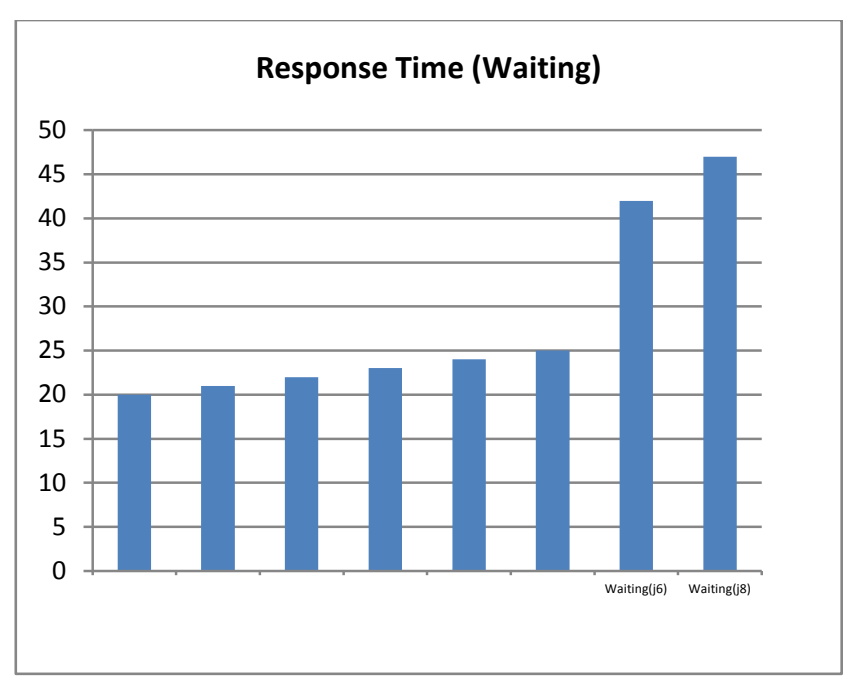

\section{Graph 1.1: Graph shows that response time reduces that} means CPU utilization improve

Graph has explained that quantized analysis of the response time. Graph clearly show that $\mathrm{j} 6$ and $\mathrm{j} 8$ at waiting state because sites did not meet requirement with available processors.

Response time (co-allocation) - If co-allocation is acceptable then job is executed with free resources in multiple clusters. Allocation is allowed with assign of important number of processors for incoming job from existing nodes

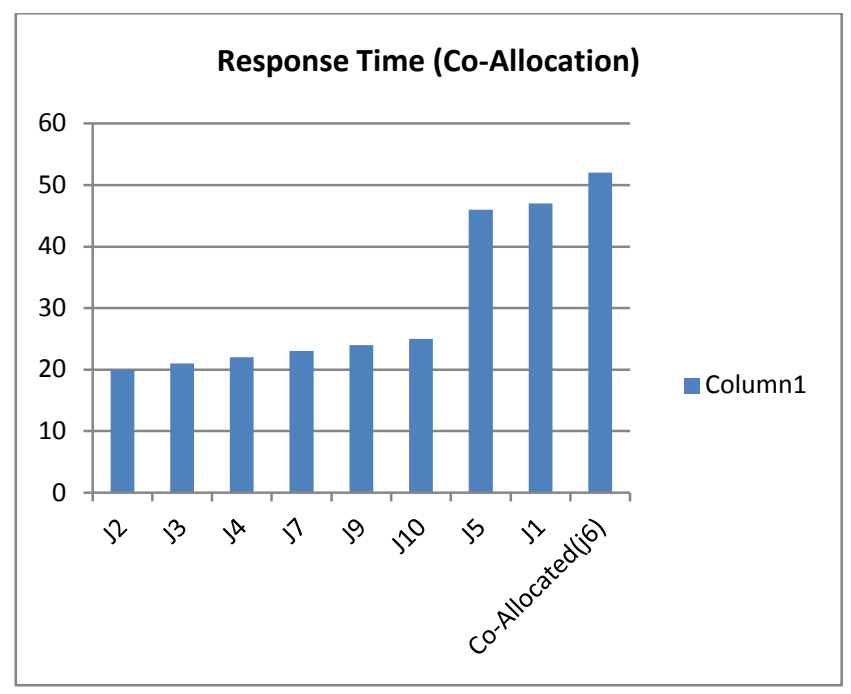

Graph 1.2: Response Time (Co-allocation)

Graph 1.2 as response time needs to be minimized; so the main goal is to decrease the response time as much as possible. It shows that co-allocation is allowed then job is executed with free resources in multiple clusters. Allocation is concerned with assignment of essential number of processors for incoming job from available sites.

\section{CONCLUSION AND FUTURE SCOPE}

In this paper we try to reduce response waiting and coallocation time when these two parameters reduce values then system performance enhanced. In future we use better performance and in further we used better scheduling policy to better improvement in System. As future work is familiar as the proposed algorithm further can be enhanced with the better scheduling policies

\section{ACKNOWLEDGMENTS}

Our thanks to my parents and my guide, Lecture who have contributed towards development of the template.

\section{REFERENCES}

[1] A.C. Kaladevi, M.V.Srinath, C.Babitha Lilly Mary, "Processor Co Allocation Enabling Advanced Reservation of Jobs using Priorities in Multi-Cluster system", international Journal of Computer Applications Volume 44,No10, pp 0975 -8887, April 2012.

[2] Dinesh Gupta, Sakshi Gupta, Neha Jhamb,"Mininum Response Time and Effective Utilization Of I/O-Bound Process Using, Approximate zero Response Algorithm", International Computer Technology \& Applications, Volume 3, No 2, pp 611-616, 2012.

[3] Héctor Blanco, Josep Lluís Lérida, Fernando Cores, Fernando Guirado "Multiple job co-allocation strategy for heterogeneous multi-cluster systems based on linear programming" The Journal of Supercomputing, Volume 58, Issue 3, pp 394-402, December 2011

[4] Huang K.C, Kuang-PO Lai 2010, "Processor allocation policies for reducing resource fragmentation in multicluster and cloud Environment", IEEE, 2010.

[5] K.C. Huang, P.C. Shih Y.C. Chang, "Effective Processor Allocation for Moldable Jobs with application speed up Model. Smart Innovation", Systems and Technologies, Vol 21, pp 563-572, 2013.

[6] K.C Huang, "Performance Evaluation of Adaptive processor allocation policies for moldable parallel batch jobs". In proceeding of the Third workshop on grid technologies and Application, 2013.

[7] Jaehwan Lee, Pete Keleher, Alan Sussman, "Decentralized dynamic scheduling across heterogeneous multi-core desktop grids", IEEE International Symposium on Parallel\& Distributed Processing, Workshops and Phd Forum, pp 1-9.2010. 